\title{
OBJECT-ORIENTED REFORMULATION AND EXTENSION OF IMPLICIT FREE-FORM DEFORMATIONS
}

\author{
Olivier Parisy, Christophe Schlick \\ LaBRI, Université Bordeaux 1, 351 cours de la Libération, 33405 Talence, France \\ (parisy|schlick)@labri.u-bordeaux.fr
}

\section{Benoît Crespin}

LIGIM, Université Lyon 1, 43 bd du 11 novembre 1918, 69622 Villeurbanne, France bcrespin@bat710.univ-lyon1.fr

\begin{abstract}
This paper proposes an extension of the Implicit Free-Form Deformation technique (IFFD, for short) recently developed by Crespin [Crespin, 1997]. The original formulation of IFFDs is based on a functional paradigm. In this paper, we show that such a functional formulation involves some limitations, especially when efficiency and extensibility are considered. To cancel these drawbacks, an object-oriented $(\mathrm{OO})$ reformulation of the technique is proposed, which leads to an efficient implementation and offers a general framework that can be used to express most of existing deformation techniques and develop some original ones.
\end{abstract}

Keywords: Geometric Modeling, Free-Form Deformations, Implicit Surfaces

\section{INTRODUCTION}

Since the innovative warping technique, introduced by Parent in 1977 [Parent, 1977], geometric deformation techniques have become ubiquitous in Computer Graphics (CG). The basic idea of deformation techniques is to put an indirection between the user and the 3D object he works on. So instead of directly editing the degrees of freedom provided by the geometric model (e.g. vertices for polygonal meshes, control points, knots or weights for spline patches), the user manipulates a deformation tool the modification of which are propagated to the model. There are at least three major advantages of deformation techniques over usual edition of 3D objects. First, understanding the meaning of the degrees of freedom provided by the geometric model (that are usually strongly 
related to its mathematical formulation) is not required. Second, the manipulation of a deformation tool is totally independent of the complexity of the object it is applied on, whereas direct edition of complex objects becomes extremely painful. Finally, as it is disconnected from the geometric model, very intuitive deformation tools can be developed, usually based on a sculpting metaphor.

These impressive features are clearly the reason for which several hundreds of research papers dealing with deformation techniques have been written during the last twenty years. Facing this multitude, a natural trend is to organize, classify and try to find a unifying framework in which all of them can be expressed. A few papers have proposed such a framework [Bechmann, 1994, Blanc et al., 1994, Crespin, 1998]. The goal of this paper is to improve one of these unifying frameworks, namely the functional paradigm, proposed by Crespin in his $\mathrm{PhD}$ thesis [Crespin, 1998], and later used to define Implicit Free-Form Deformations (IFFD) [Crespin, 1997].

The remainder of the paper will be organized as follows. Section 2 recalls some previous work (free-form deformations, combination of deformations, implicit surfaces) needed to understand the IFFD model. Section 3 describes the original IFFD framework, as presented in [Crespin, 1997], and exhibits some of its limitations. Section 4 proposes an object-oriented reformulation of IFFDs that cancels the previous limitations. Finally, Section 5 presents some results while Section 6 concludes and proposes some directions for future work.

\section{PREVIOUS WORK}

A general presentation of deformation techniques is out of the scope of this paper (see [Bechmann, 1994, Güdükbay and Özgüç, 1990, Mikita, 1996] for an almost exhaustive survey). This section only aims at exhibiting two of their typical features, the concept of local coordinates to express deformation tools and the idea of building complex deformations by composing simpler ones. Two families of deformations (Free-Form Deformations and Constraint-based Deformations) will be presented for this purpose. The basics of implicit objects will also be recalled, as they are the kernel of the IFFD technique.

\subsection{FREE-FORM DEFORMATIONS}

Looking at its usage and its numerous derivatives, Sederberg and Parry's Free-Form Deformation (FFD) [Sederberg and Parry, 1986] is probably one of the most successful deformation tool. We will only describe here the original FFD technique, but a whole succession of tools have evolved from this first formulation. It is based on the following mechanism :

1 a lattice (a parallelepipedic network) surrounding the object to deform is defined with a given number of subdivisions following the three coordinates axis (Figure 1, on the left) ; 
2 for each point of the object, its local coordinates relative to the lattice (called lattice coordinates) are computed ;

3 points of the lattice are then moved by the user ;

4 for each point of the object, its new global coordinates are computed, considering that the set of points of this lattice defines a Bézier volume (using a trivariate tensor product), and that the lattice coordinates have not changed (Figure 1, on the right).

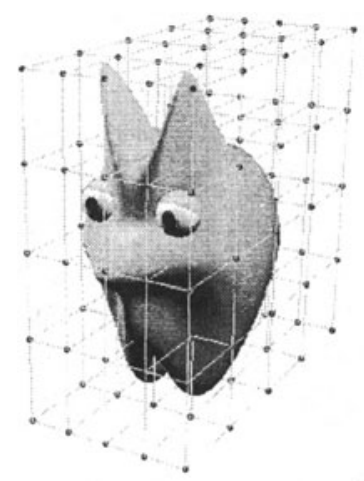

Initial object and lattice

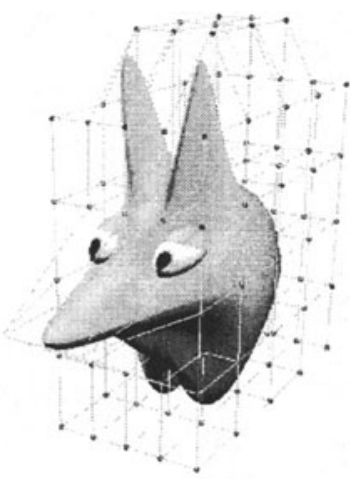

Final object and lattice

\section{Figure 1 An FFD example}

The first step corresponds to the definition and the location of the deformation tool, the second one to a freezing of the coordinates, the third to the manipulation of the tool and the fourth to an unfreezing of coordinates, resulting in a globalspace deformation.

This technique has some very interesting features :

- The fact that the deformation is computed with the hypothesis that the lattice coordinates are frozen leads to a rather intuitive behavior : from a user's point of view, the object seems to be embedded in a transparent jelly (the lattice), which communicates its deformation to the model.

- The freezing step, which is the most computation demanding, is done only once (its result is kept during the whole interaction) whereas the local deformation and the unfreezing, much less expensive, are usually done in an interactive way.

- By moving only a few points of the lattice, a continuous deformation of an arbitrary number of points of the model is obtained. From this point of view, FFD may be referred to as a "high-level" deformation tool. 
Note that the freezing step is discrete in nature; because its result has to be cached, it can only be performed on a finite subset of characteristic points of the object (cf. Section 3.2).

\subsection{CONSTRAINT-BASED DEFORMATIONS}

Another interesting deformation technique was proposed by Borrel and Rappoport [Borrel and Rappoport, 1994] and can be seen as a generalization to continuous deformations of the original warping technique developed by Parent [Parent, 1977]. In this approach, a set of $n$ displacement constraints are simultaneously applied to a volume. Each constraint is defined as an ellipsoid $C_{i}(i \in[1, n])$ with a center $O_{i} \in \mathbb{R}^{3}$ and a weighting function $F_{i}: \mathbb{R}^{3} \rightarrow \mathbb{R}$ that is inversely proportional to the distance from $O_{i}$.

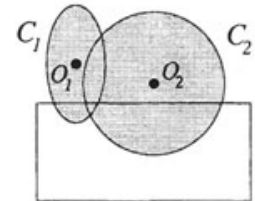

Initial situation

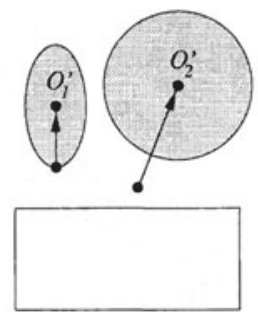

Constraints displacement

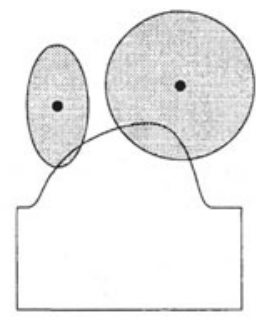

Deformation

Figure 2 Constraint-based deformation scheme

The first step in the deformation process lets the user place the ellipsoids at their initial location $O_{i}$. Once the user has translated one or more ellipsoids to some new locations $O_{i}^{\prime}$, a displacement is applied to any point $M \in \mathbb{R}^{3}$. This displacement is roughly defined as the sum of the local displacements $\overrightarrow{O_{i} O_{i}^{\prime}}$, weighted by the values $F_{i}(M)$. This process is summarized on Figure 2 .

More precisely, the resulting deformation $D$ from $\mathbb{R}^{3}$ to $\mathbb{R}^{3}$ is given as a $3 \times n$ matrix $D_{m}$ which must satisfy Equation $1 . D_{m}$ is obtained by a pseudoinversion algorithm depending on the initial positions $O_{i}$ and the weighting functions $F_{i}$.

$$
\forall i \in[1, n], O_{i}^{\prime}=D\left(O_{i}\right)=D_{m} \times\left[\begin{array}{c}
F_{1}\left(O_{i}\right) \\
F_{2}\left(O_{i}\right) \\
\ldots \\
F_{n}\left(O_{i}\right)
\end{array}\right]
$$

This approach, called Scodefs for "Simple Constrained Deformations", offers an arbitrary combination of global or local deformations, especially because it is 
not dependent on a particular topology as are traditional FFD. Moreover, Bechmann [Bechmann, 1994] showed that FFD can more or less be reformulated in the Scodefs formalism.

But this technique also has serious drawbacks. First, because the matrix computation is performed after each displacement of an ellipsoid, the freezing step of traditional FFD is lost, and this forbids the use of too many constraints. Scodefs are also poorly intuitive when constraints are too close from each other. Finally, specific kinds of deformations such as twisting or tapering are impossible to obtain with Scodefs unless allowing rotation or scaling of ellipsoids in addition of translation ; unfortunately, the computation of the deformation matrix is much more complicated in this case.

\subsection{IMPLICIT OBJECTS}

The idea of using implicit models in geometric modeling was initially proposed by Blinn [Blinn, 1982] (see [Bloomenthal et al., 1997] for an exhaustive survey). An implicit object is based on a set of primitives $P_{i}$ where each primitive is the source of a potential field, defined by a field function $F_{i}(x, y, z)$ that maps $\mathbb{R}^{3}$ to $\mathbb{R}$ (or a subset of $\mathbb{R}$ ). At a given point $P(x, y, z)$ of the Euclidian space, the fields of all the sources are computed and added together (blending), leading to a global field function $F(x, y, z)$ :

$$
F(x, y, z)=\sum_{i=1}^{n} F_{i}(x, y, z)
$$

A 3D surface can then be defined from this global field function $F(x, y, z)$ by giving a threshold value $T$ and rendering the equipotential surface $S$ for this threshold :

$$
S=\left\{(x, y, z) \in \mathbb{R}^{3} / F(x, y, z)=T\right\}
$$

The advantage of this approach is that union or difference of primitives are then equivalent to sums or differences of their associated potentials, which are straightforward to perform. It is thereof easy to combine an arbitrary number of simple primitives to obtain a complex one. If correct hypothesis on potentials are fulfilled, the resulting surface is continuous and, unlike other models, no intersection computation is needed to generate the global surface.

Though this approach exhibits seductive properties, some trade-off must be highlighted :

- Implicit surfaces are difficult to visualize in an interactive way because the tessellation needed to get hardware rendering is expensive [Bloomenthal, 1994] ; 
- Texture mapping is difficult to apply to implicit surfaces as they do not exhibit a natural parameterization.

It must be noticed that these drawbacks only apply if implicit objects are used for geometric modeling. If they are considered as a mathematical model for doing anything else, interactive visualization and texture mapping are not required anymore and only the good side of the model is kept.

\section{IMPLICIT FREE-FORM DEFORMATIONS}

\subsection{MOTIVATION}

As noted by Crespin [Crespin, 1998], the weighting approach found in Scodefs is very similar to the blending process used in implicit-based modeling. Weighting functions $F_{i}$ (cf. Section 2.2) actually look like usual field functions found in implicit literature, although the authors do not refer to them. Consequently, these functions permit an automatic continuity on the boundary between different deformation constraints, which recalls the continuity provided between blended implicit primitives. Moreover, the results obtained by combining several deformations are often prone to unwanted artefacts (bulges, lost of symmetry, non-commutative combinations, etc.) that can only be reduced by complex and non-intuitive tricks (direct manipulation of control points, use of additional deformations, etc.). An unified tool to express the combination of deformations may be a solution to cancel these artefacts. Hence, he proposed a deformation technique called Implicit FFD (IFFD) which :

- Allow the combination of different deformation primitives according to some field (or weighting) functions associated to each one. This combination would ideally be simpler than in the Scodef model.

- Provide high-level deformation primitives, based on local coordinates systems, such as FFD tools. Indeed, a 3-step recurrent scheme (freezing, manipulation, unfreezing) can be seen in the way many deformation tools are used. Thus, it seems interesting to formalize these tools so that they may be manipulated in an unified way.

- Re-introduce a freezing step to avoid expensive computations.

\subsection{DEFORMATION FUNCTION}

In most cases, a deformation function $D$ will be applied to discrete sets of characteristic points (such as vertices of polygonal meshes, control points of spline patches, etc.) which will be represented as a vector $\vec{M}$ of size $m$ (its components $M_{i}$ being the points). This will abusively be referred to as "points deformation" or "coordinates deformation", though it only defines the 
translation of each $M_{i}$ to $M_{i}^{\prime}=D\left(M_{i}\right)$. Similarly, we will also often refer to "points" where "coordinates of points" would be more appropriate.

A local space $\mathbb{L}$ can be associated to each deformation tool ; in most cases, it will be $\mathbb{R}^{d}$, with $d \leq 3$. The global deformation function $D$ can then be decomposed in

- $\phi\left(\mathbb{R}^{3} \rightarrow \mathbb{L}\right)$ : the (invertible) immersion function ;

- $\Delta(\mathbb{L} \rightarrow \mathbb{L})$ : the local space deformation.

Where $D=\phi^{-1} \circ \Delta \circ \phi$ and

$$
M_{i} \stackrel{\phi}{\rightarrow} \tilde{M}_{i} \stackrel{\Delta}{\rightarrow} \tilde{M}_{i}^{\prime} \stackrel{\phi^{-1}}{\longrightarrow} M_{i}^{\prime} .
$$

In this formulation, the superscript " " " means local coordinates and " 1 " means deformed coordinates ; $\phi$ is the freezing step, $\Delta$ the manipulation step and $\phi^{-1}$ the unfreezing step already described in Section 2.1.

As an example, an FFD with an initial lattice $L$ and a deformed lattice $L^{\prime}$ can be expressed as follows :

$$
\left(M_{i}, L\right) \stackrel{\phi}{\rightarrow}\left(\tilde{M}_{i}, L\right) \stackrel{\Delta}{\rightarrow}\left(\tilde{M}_{i}, L^{\prime}\right) \stackrel{\phi^{-1}}{\longrightarrow}\left(M_{i}^{\prime}, L^{\prime}\right),
$$

In other words, we consider the coordinates used by the deformation process as couples composed of a characteristic point $M_{i}$ and a lattice $L$.

\subsection{DEFORMATION PRIMITIVES}

A deformation primitive $\Pi_{j}$ is defined as a global deformation fonction $D_{j}$ associated to a scalar field function $F_{j}$ of the same kind of those used in implicit objects (see Section 2.3).

When the primitive $\Pi_{j}$ is applied on a given point $M_{i}$, it defines a couple $\left(d_{i j}, f_{i j}\right)$ where $d_{i j}=D_{j}\left(M_{i}\right)$ (the point after deformation) and $f_{i j}=F_{j}\left(M_{i}\right)$ (its associated scalar field value).

By applying $n$ primitives $\Pi_{j}$ to $m$ points $M_{i}$, we define a $m \times n$ deformation matrix $\mathcal{D}$ :

$$
\mathcal{D}=\left[d_{i j}\right] \text { where } d_{i j}=D_{j}\left(M_{i}\right)
$$

and a $m \times n$ field matrix $\mathcal{F}$ :

$$
\mathcal{F}=\left[f_{i j}\right] \text { where } f_{i j}=F_{j}\left(M_{i}\right)
$$

\subsection{BLENDING FUNCTION}

A blending function $\Psi$ is defined to collapse each row of matrix $\mathcal{D}$ into a single value $M_{i}^{\prime}$ that represents the final image of $M_{i}$. This blending is a $n$-ary operator that uses the corresponding rows of matrix $\mathcal{F}$ as weighting factors. 
A possibility for $\Psi$ that behave nicely (commutative, $I_{\mathbb{R}^{3}}$ when no primitive is modified) is :

$$
M_{i}^{\prime}=M_{i}+\frac{\sum_{j=1}^{n} F_{j}\left(M_{i}\right)\left(D_{j}\left(M_{i}\right)-M_{i}\right)}{\sum_{j=1}^{n} F_{j}\left(M_{i}\right)} .
$$

\subsection{IFFD}

Finally, by collecting all previous components, an IFFD is defined by a set of $n$ deformation primitives and a blending function $\Psi$. When applied to a point vector $\vec{M}$, the resulting point vector $\vec{M}^{\prime}$ is defined by :

$$
\vec{M}^{\prime}=\Psi(\mathcal{D}(\vec{M}), \mathcal{F}(\vec{M}))
$$

Figures 3 presents an example of a simple IFFD that involves two spherical primitives and a translation of one of them. It is worth noticing that even such simple instances of IFFDs are able to produce effects similar to Barr's classical bend or twist operators [Barr, 1984].

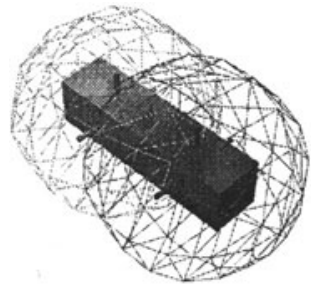

Locating primitives

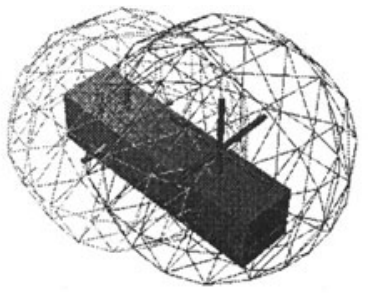

Translating a primitive

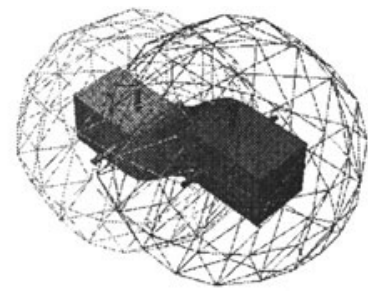

Final deformation

Figure 3 Translation of a primitive

\section{OBJECT-ORIENTED FORMULATION OF IFFD}

The formulation of the FFD given in Section 3.2 shows that a strictly functional representation, even if expressive enough, may get somewhat bloated. Indeed, as it manipulates couples composed of a point $M_{i}$ and a lattice $L$, the lattice has to be duplicated at $m$ occurrences to fulfill the functional paradigm.

Moreover, this formulation is not able to manage two other points of practical interest :

- The freezing step (done once) is clearly distinct from the unfreezing (done at an interactive rate). 
- The user has no direct access to the local coordinates (i.e. relative to the deformation tool) which are always internal to the deformation function.

To address all these problems, we propose to reformulate IFFD by using an object-oriented (OO) approach.

\subsection{OO REFORMULATION OF DEFORMATIONS}

Computations for all components of a point vector $\vec{M}$ can be combined : instead of considering $D$ as a function from $\mathbb{R}^{3}$ to $\mathbb{R}^{3}$, and then extending it to point vectors by applying it to each component of this vector, we will now define it as an algorithm taking these vectors as parameters from the start; this way, more efficient computation may be performed.

More precisely, a deformation can then be defined as a class affording the following methods :

\begin{tabular}{cccc}
\hline Method & Parameters & Behavior & Return value \\
\hline freeze & $\vec{M}$ & Computes $\tilde{M}_{i}$ & None \\
manip & & Computes $\tilde{M}_{i}^{\prime}$ & None \\
unfreeze & None & None & $\vec{M}^{\prime}$ \\
\hline
\end{tabular}

Note that by using methods, we have dropped the concept of mathematical function, because border effects are now possible. Indeed, the local coordinates $\tilde{M}_{i}$ (before tool manipulation) and $\tilde{M}_{i}^{\prime}$ (after tool manipulation) are now local attributes of the deformation class, so that they can only be manipulated by its methods. The idea here is that because the local space is specific to each deformation tool, hiding them leads to an unified vision (and manipulation) of them.

\subsection{EXTENSION TO THE IFFD MODEL}

Using the OO-formulation of deformations, so that each primitive $\Pi_{j}$ encapsulates objects of this class, IFFD can be reformulated as specializations of the same class :

\begin{tabular}{|c|c|c|c|}
\hline Method & Parameters & Behavior $(\forall j)$ & Returns \\
\hline freeze & $\vec{M}$ & $\left\{\begin{array}{l}\text { Computes } \mathcal{F} \\
\Pi_{j} . \text { freeze }(\vec{M})\end{array}\right.$ & None \\
\hline $\begin{array}{l}\text { manip } \\
\text { unfreeze }\end{array}$ & None & $\begin{array}{c}\Pi_{j} \cdot \text { manip }() \\
\Pi_{j} \cdot \text { unfreeze }() \rightarrow \mathcal{C}\end{array}$ & $\begin{array}{l}\text { None } \\
\Psi(\mathcal{C}, \mathcal{F})\end{array}$ \\
\hline
\end{tabular}


It is interesting to notice that IFFDs themselves can be expressed in the same formalism as the deformations which compose them; this illustrates their genericity and the adequacy of our formulation.

\section{SOME RESULTS}

As a proof of concept, and to test IFFDs usability, this OO-framework was implemented as a plug-in for Maya (the 3D modeling and rendering software environment developed by Alias|Wavefront [Alias|Wavefront, 2000]). We will present here some meaningful use of this tool.

\subsection{BASIC EXAMPLES}

The following pictures will illustrate the various degrees of liberty available using IFFDs. In all of them, only simple affine transformations are used for the manipulation of the primitives. But of course, more complex manipulations may be used.

Figure 4 shows two spherical primitives located on a plane ; by applying rotations, translations and scalings to these primitives, the plane is accordingly deformed. Notice how influence of the two primitives blend smoothly in the zone deformed by both primitives (i.e. in the intersection of the balls defining non-null fields). The deformation depends also on the geometry of the field (Figure 5) and of the way it decreases (Figure 6). Finally, a more complete example is presented on Figure 7.

\subsection{MORE DETAILED EXAMPLES}

More complex objects were modeled to test whether interesting shapes could be achieved. As an example, Figure 8 shows a ship and a little creature (notice the spherical-shaped primitive bending its ear) built by performing various IFFD-based deformations on an initial sphere. Note that for all primitives, the deformation functions used were always combinations of translations, rotations and scalings (as for the examples of Section 5.1). Spherical and conic fields were used and combined to a Blanc-Schlick function [Blanc and Schlick, 1995] to profile their decreasing.

As an illustration, Figure 9 details the ship's noozle hollowing. A single conic primitive was used for this purpose : after the creation and setting of the primitive (left picture), it was translated to the front of the hull, which resulted in the expected hole (right picture). 

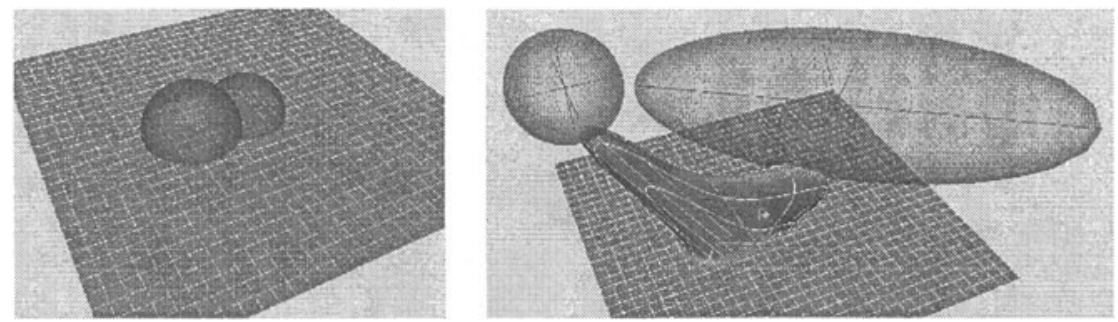

Figure 4 Deforming a plane using two primitives
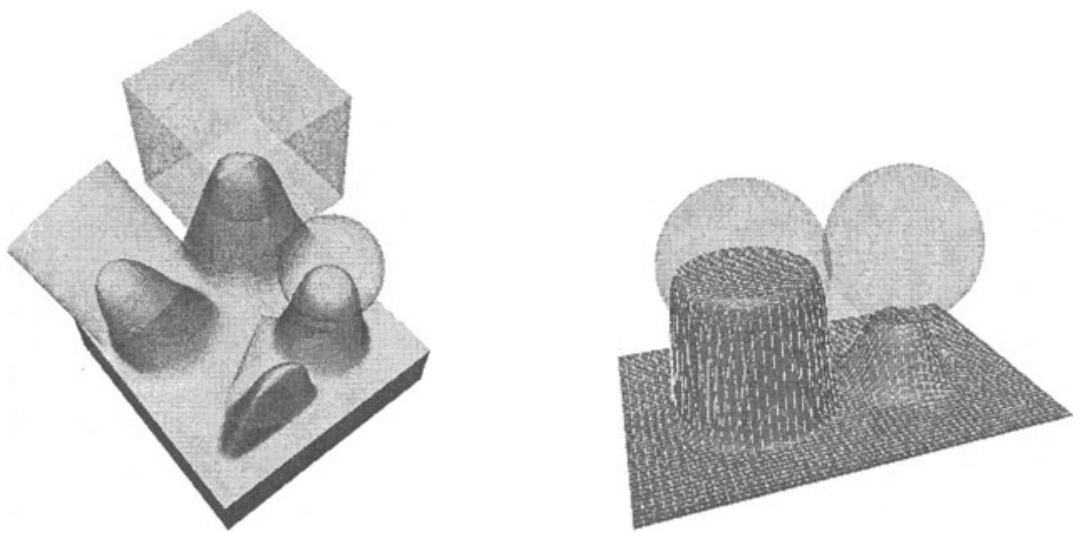

Figure 5 Miscellaneous field shapes

Figure 6 Some field decreasings
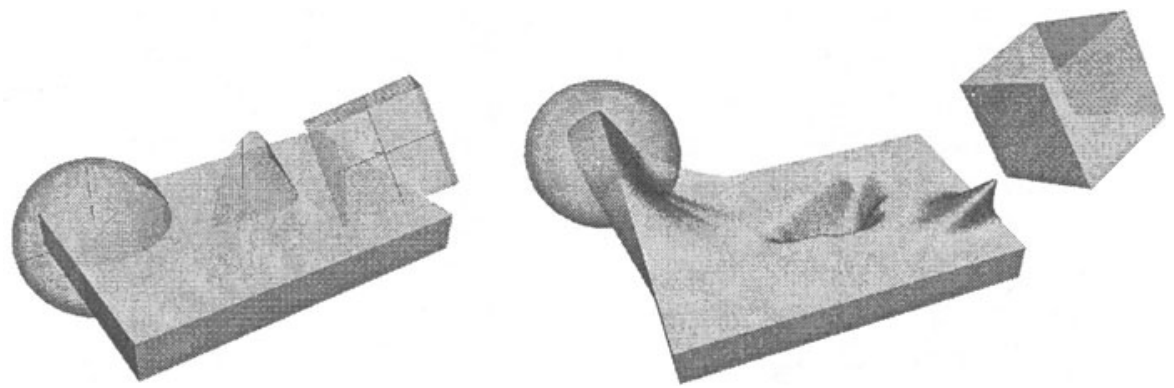

Figure 7 Complete IFFD example 

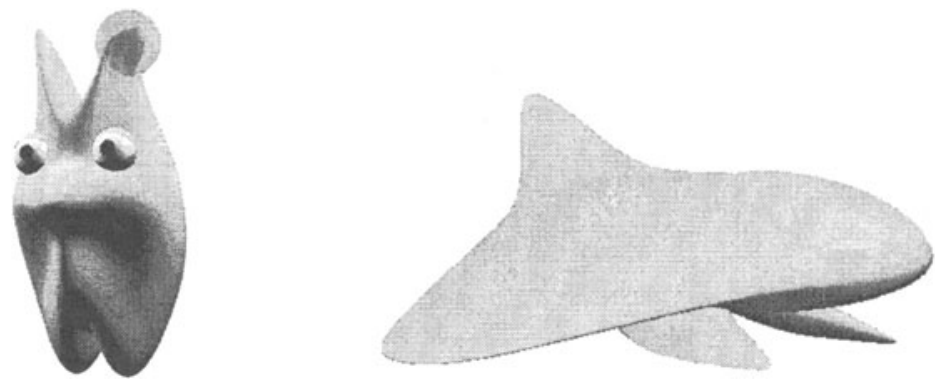

Figure 8 Models designed using IFFDs

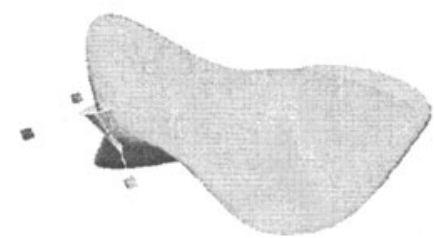

Initial setting of the primitive

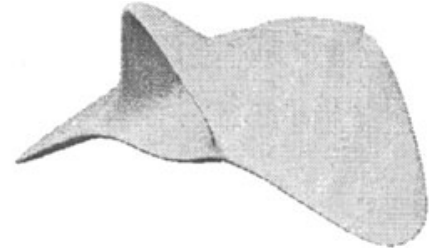

After primitive translation

Figure 9 Nozzle hollowing using an IFFD

\section{CONCLUSION}

After a presentation of some interesting trends in free-form deformation and how IFFD unifies most of them, we showed how its object-oriented formulation expresses well their modularity and the way they are used.

This leads to a straightforward implementation in the data flows paradigm of Maya, which proved useful even with simple primitives because of the various degrees of liberty permitted by the many field parameters. Interestingly, these can be easily manipulated using a GUI, which enabled the geometric modeling of complex objects as showed in Section 5.2.

A possible extension would be to include more complex scalar fields ; generalized cylinders as an example may be used. Another idea would be to test whether interesting animations could be obtained using fields changing over time or objects moving in those fields, as with AFFD [Coquillart and Jancéne, 1991].

\section{References}

[Alias|Wavefront, 2000] Alias|Wavefront (2000). (http://www. aliaswavefront. com/en/Home/homepage.html). 
[Barr, 1984] Barr, A. (1984). Global and local deformations of solid primitives. Computer Graphics (SIGGRAPH'84 Proceedings), 18(3):21-31.

[Bechmann, 1994] Bechmann, D. (1994). Space deformation models survey. Comput. \& Graphics, 18(4):571-586.

[Blanc et al., 1994] Blanc, C., Guitton, P., and Schlick, C. (1994). A methodology for description of geometrical deformations. In Pacific Graphics'94.

[Blanc and Schlick, 1995] Blanc, C. and Schlick, C. (1995). Extended Field Functions for Soft Objects. In Implicit Surfaces'95 Proceedings, pages 2132.

[Blinn, 1982] Blinn, J. (1982). A generalization of algebraic surface drawing. Trans. on Graphics, 1(3):235-256.

[Bloomenthal, 1994] Bloomenthal, J. (1994). An implicit surface polygonizer. In Graphics Gems, volume 4, pages 324-349.

[Bloomenthal et al., 1997] Bloomenthal, J., Bajaj, C., Blinn, J., Cani-Gascuel, M.-P., Rockwood, A., Wyvill, B., and Wyvill, G. (1997). Introduction to Implicit Surfaces. Morgan Kaufmann.

[Borrel and Rappoport, 1994] Borrel, P. and Rappoport, A. (1994). Simple constrained deformations for geometric modeling and interactive design. ACM Transactions on Graphics, 13(2):137-155.

[Coquillart and Jancéne, 1991] Coquillart, S. and Jancéne, P. (1991). Animated free-form deformation: An interactive animation technique". In $S I G$ GRAPH '91 Proceedings, pages 23-26.

[Crespin, 1997] Crespin, B. (1997). Implicit free-form deformations. In Implicit Surface 99 Proceedings, pages 151-158.

[Crespin, 1998] Crespin, B. (1998). Modélisation et déformation de forme libre à base de surfaces implicites équipotentielles. $\mathrm{PhD}$ thesis, Université Bordeaux I.

[Güdükbay and Özgüç, 1990] Güdükbay, U. and Özgüç, B. (1990). Free-form solid modeling using deformations. Comput. \& Graphics, 14(3/4):491-500.

[Mikita, 1996] Mikita, M. (1996). 3d free-form deformation: Basic and extended algorithms. In Purgathofer, W., editor, Proc. of the $12^{\text {th }}$ Spring Conference on Computer Graphics, pages 183-191. Comenius University, Bratislava.

[Parent, 1977] Parent, R. (1977). A system for sculpting 3-d data. Computer Graphics, 11(2):138-147.

[Sederberg and Parry, 1986] Sederberg, T. and Parry, S. (1986). Free-form deformations of solid geometric models. Computer Graphics (SIGGRAPH'86 Proceedings), 20(4):151-160. 